\title{
Social Media for the Surgeon
}

\author{
Omar Faruque Yusuf ${ }^{*}$ \\ 'Department of Surgery \\ Chittagong Medical College \\ Chattogram, Bangladesh.
}

*Correspondence to:

Professor (Dr.) Omar Faruque Yusuf

Professor \& Head (Retired)

Department of Surgery

Chittagong Medical College

Chattogram, Bangladesh.

Mobile : +8801711750678

Email : ofyusuf@yahoo.co.uk

Date of Submission : 02.06 .2021

Date of Acceptance : $\quad 30.06 .2021$
In 2004 we entered the World Wide Web 2.0 age. Almost overnight, two-way communication became a reality and content creation became available to anyone. These technologic advances led to bidirectional communication where users could not only comment on content, but also edit and develop their own. It seemed everyone had an equal voice, whether good or bad, truthful or not. As time went on, these websites incorporated a strong social component, and encouraged user-generated content in the form of text, video, photo postings, and blogs, all of which can occur instantaneously. Out of this social media was bornwith sites like Facebook, Twitter, Linkedin, Instagram, Whatsapp, YouTube and Snapchat to name a few.

Worldwide, there are over 2.8 billion monthly active Facebook users, 192 million Daily Active Twitter Users, YouTube registers over 300 hours of video uploaded every minute and almost 5 billion videos are watched every single day.In Bangladesh, there are currently over 40 million Facebook users. Doximity, a US based physicianonly social network started in 2011, where physicians can use their real name, contact others and verify credentials has over 800,000 Licensed Medical Professionals and in less than six years, approximately 70 percent of all doctors in the US have joined Doximity to connect, collaborate and collectively provide better care to their patients.

Surgeons are no different than the general population in adopting new technologies such as social media for their personal use. YouTube videos demonstrate multiple surgical techniques, all with the click of a mouse. Residents, fellows and fully trained surgeons alike have reported on regular use for both educational and "brushup" purposes.

Yet, these are simply the beginnings and we are currently in the infancy of their use. Professionally, surgeons in particular, have embraced this technology with hesitancy and trepidation - perhaps due to busy schedules and or lack of computing skills.. But the reality remains: If you ignore your blog or social media site, or lack one altogether, you may be perceived out of touch or disinterested. The educational opportunities for social media are endless, as clinicians in remote settings now can have access to tertiary care centers and expert opinions via telemedicine and telementoring.Currently,leading Surgical Associations and societies have incorporated Facebook, Twitter and YouTube into their national meetings and websites. However, it is not without vulnerability.

Professionalism is the basis of a physician's contract with society. It mandates integrity, accountability and the continued pursuit of excellence. Although these standards are well known and well accepted in clinical practice, the lines can be blurred in the anonymity of the Internet. Medical professionals can find it difficult to apply the principles of professionalism to online communication. They need to consider the impact of their online content on the public and ensure that postings do not disregard 
patient privacy or create unprofessional content online including plagiarism,that can reflect poorly on the entire profession. Hence, it is important to understand the limitations and use of the Internet platforms' privacy and intellectual property rights settings. Fake news, fake postings, cybersecurity, are all painful reminders of the fragile susceptibility of the internet.

With that in mind, the American Medical Association Council of Ethical and Judicial Affairs have published a policy on Professionalism in the Use of Social Media. Although these recommendations may seem self-evident to some and draconian and out of touch to others, they do offer a framework to help physicians navigate the Internet and social media world while maintaining their professionalism. The number one priority is obvious - maintain patient privacy and confidentiality.

The world was ill prepared to deal with the COVID 19 pandemic in 2020. The consequent horrific fallout in terms of worldwide mortality and morbidity,countrywide lockdowns, closure of educational institutions, record numbers of unemployment, collapse of national health services along with the travel-tourism-restaurant-leisure industry brought the world to the brink of a recession and depression not seen since the 1930's and the Second World War. The world changed and had to adapt to the new order. With the pandemic raging and people confined to their homes, Video conferencing apps like Zoom, Cisco Webex Meetings, Skype MeetNow, Microsoft Teams, Discord and
Google Hangouts allowed us to stay in touch and became central to our day-to-day lives. Online and social media boomed as the populace had to work remotely from home; education and research continued with web-based teaching and conferencing platforms.Currently Zoom pulls in more than 200 million daily active users. Webinars, Online and hybrid conferences are now well accepted, though lesser alternatives to the traditional live sessions. These meetings have the convenience of being able to participate from any corner of the world, at any time, without the discomforts of travel, formal dress codes, hotel bookings and other logistics. However interpersonal relationships suffer in the absenceof relaxed discussions of a professional and personal nature over a cup of coffee or a warm meal with old or new colleagues.

A COVID 19 free world or at least a pandemic controlled one is a utopian idea and vaccination of the world populace a hopeful distant vision. The world as we knew it before the advent of the pandemic may never return to normal soon. Social networking has gone on for generations with family, friends and colleagues via handwritten letters, analog and mobile telephones and now smartphones and smartpads. Post pandemic, in the now recognized "new normal", with each technological advance, information is being disseminated faster, easier, and sometimes even better. To better inform and care for patients it is important that we embrace these advances.

\section{REFERENCES}

1. Social Media and the Surgeon - NCBI - NIH.

https://www.ncbi.nlm.nih.gov/pmc/articles/PMC3699141/by DA Margolin - 2013.

2. Goldman D Facebook tops 900 million users.

Available at: http://money.cnn.com/2012/04/23/technology/facebook-q1/index.htm

3. You Tube Available at: http://www.youtube.com/t/faq.

Accessed June 26, 2012.

4. Leow J J, Pozo M E, Groen R S, Kushner A L. Social media in low-resource settings: A role for Twitter and Facebook in global surgery? Surgery. 2012;151(6):767-769.[PubMed].

5. Bennett E Found in cache. Social media resources for health care professionals. http://ebennett.org/hsnl/.

6. Greysen S R, Kind T, Chretien K C. Online professionalism and the mirror of social media. J Gen Intern Med. 2010;25(11):1227-1229. [PMC free article] [PubMed].

7. Lesse C S, Lucey C R, Egner B et al. A behavioral and systems view of professionalism. JAMA. 2011;304:2732-2737. [PubMed].

8. Thompson L A, Dawson K, Ferdig R et al. The intersection of online social networking with medical professionalism. J Gen Intern Med. 2008;23(7):954-957.[PMC free article] [PubMed].

9. AMA Council on Ethical and Judicial Affairs . Professionalism in the Use of Social Media. J Clin Ethics. 2011;22:97-104. [PubMed]. 